The Astrophysical Journal, 331 :949-957, 1988 August 15

(C) 1988. The American Astronomical Society. All rights reserved. Printed in U.S.A

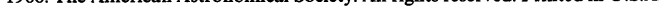

\title{
EXOSAT OBSERVATIONS OF THE CASSIOPEIA A SUPERNOVA REMNANT
}

\author{
F. JANSEN \\ Laboratory for Space Research, Leiden \\ A. SMITH \\ Space Science Department, ESTEC, Noordwijk \\ J. A. M. BLEEKER \\ Laboratory for Space Research, Utrecht \\ P. A. J. DE KORTE ${ }^{1}$ \\ Laboratory for Space Research, Leiden \\ AND \\ A. Peacock And N. E. White \\ Space Science Department, ESTEC, Noordwijk \\ Received 1986 December 16; accepted 1988 February 10
}

\begin{abstract}
The young supernova remnant (SNR) Cas A has been observed with the European X-ray observatory satellite EXOSAT. The remnant was observed with all instruments covering the range from 0.5 to $25 \mathrm{keV}$.

It is shown here that the emission from the remnant cannot be described by a two-temperature isothermal plasma in collisional ionization equilibrium.

A simple Sedov blast-wave model assuming equilibration between the electron and ion temperature but nonionization equilibrium (NIE) also does not result in an acceptable fit due to strongly enhanced emission at low energies $(\leq 2.5 \mathrm{keV})$. Observations made with the EXOSAT imaging proportional counter show that this extra emission comes from the interior regions of the remnant. The source of this low-energy flux is most probably supernova ejecta heated in a reverse shock.
\end{abstract}

No evidence is found for a high temperature $(\geq 20 \mathrm{keV})$ component.

Subject headings: nebulae: individual (Cas A) - nebulae: supernova remnants — radiation mechanisms X-rays: sources

\section{INTRODUCTION}

Cas $\mathrm{A}$ is one of the most well-studied young supernova remnants (SNRs). The supernova itself was probably observed by Flamsteed in 1680 indicating an age of 307 years. This agrees quite well with the value for the explosion date of $1658 \pm 3$ measured from the expansion of the optical filaments (van den Bergh and Kamper 1983). The situation is different at radio wavelengths where a larger age is deduced from proper motions of radio knots ( $\sim 950 \mathrm{yr}$, Tuffs 1986); however, the relationship between average apparent expansion and age is less certain in the radio region. A comparison between radial velocities and proper motions of the optical filaments yield a measure of the distance of $2.92 \mathrm{kpc}$ (Braun 1985).

The optical filaments in Cas A can be divided into a system of fast moving knots (FMK) and quasi-stationary flocculi. The FMK show an overabundance of oxygen, argon, and calcium but no evidence of emission from hydrogen, helium, iron, and magnesium (see, e.g., Chevalier and Kirshner, 1978). This is suggestive of the ejecta from a relatively massive star, indicating a Type II SN. Cas A lies in the approximate line of sight and at a comparable distance to the Cas OB2 association which, if genuinely linked, would support the theory of a massive progenitor.

The observations of SNRs in general reached a milestone with the detection of an iron emission line in Cas A (Serlemitsos et al. 1973). Since then a large number of X-ray observations have added to our knowledge. Early studies lands.
(Charles et al. 1975; Davison, Culhane, and Mitchell 1976) indicated a two-component thermal spectrum with components in the temperature ranges $3.5-5 \mathrm{keV}$ and $0.7-1 \mathrm{keV}$ assuming collisional ionization equilibrium (CIE). A reverse shock model (McKee 1974) with two physical components (shock and reverse shock) was suggested (Itoh 1977). Subsequently, the $H E A O 1 \mathrm{~A} 2$ experiment measured X-rays with energies up to $25 \mathrm{keV}$ and inferred temperatures greater than $\sim 10^{8} \mathrm{~K}$ (Pravdo and Smith 1979). The presence of these highenergy photons was interpreted as evidence that non-Coulomb processes dominate the heating process of the electrons (McKee 1974).

The Einstein Observatory studied Cas A with a range of instrumentation, produced high-resolution images (HRI, Fabian et al. 1980), spatially resolved low-resolution spectral data (IPC, Murray et al. 1979) and high-resolution spectral data (SSS, Becker et al. 1979; FPCS, Markert et al. 1983). The solid state spectrometer showed lines due to $\mathrm{Si}, \mathrm{S}, \mathrm{Ca}$, and $\mathrm{Ar}$ for the first time. Becker et al. fitted a two-component CIE model with temperatures of $0.65 \mathrm{keV}$ and $4 \mathrm{keV}$; the latter was fixed because of the relatively narrow band width of the SSS. The elemental abundances, which were free parameters, were found to be generally enhanced compared wih solar except for the iron, deduced from the Fe-L complex between 0.8 and 1.2 $\mathrm{keV}$, which was found to be at 0.8 solar.

The X-ray image observed with the HRI suggested that the emission arose from two thin concentric shells, again supporting the idea of a reverse shock model. An outer halo of $6^{\prime}$ radius has been detected in the HRI image (Stewart, Fabian, and Seward 1983) but the physical association with the remnant is unclear. 
It has become evident from both observations and theoretical studies that the X-ray emitting plasma in young SNRs is probably not in CIE (i.e., nonionization-Equilibrium = NIE) and a number of authors have attempted to make model predictions for SNRs in this condition (e.g., Itoh 1977; Hamilton, Sarazin, and Chevalier 1983). In these models usually a Sedov phase is assumed. The apparent over abundance of $\mathrm{Si}, \mathrm{S}$, etc. is at least partially explained in this way. Pravdo and Nugent (1983) have examined the combined SSS and HEAO 1 A2 data in the context of an NIE model and produced the requirement for two components. Itoh and Fabian (1984) examined the $\mathrm{Fe} / \mathrm{Ni}$ emission line complex seen in the HEAO $1 \mathrm{~A} 2$ data and deduced that the plasma in Cas A was in an anomalously high ionization state.

Gas scintillation proportional counters (GSPCs) provide relatively good spectral resolution over a reasonably broad energy range. Tenma observed Cas A wih GSPCs in the range $2-12 \mathrm{keV}$ and observed the emission features due to $\mathrm{S}, \mathrm{Ar}, \mathrm{Ca}$, and Fe (Tsunemi et al. 1985). The Tenma results were consistent with a single-component X-ray emitting plasma in NIE. The results were interpreted according to the model of X-ray production by a reverse shock moving into the processed supernova ejecta.

We have observed Cas A with EXOSAT and obtained useful data from all of the experiments on board. The GSPC provides a high-resolution spectrum in which the usual emission features mentioned above are observed. The medium energy (ME) experiment with its higher sensitivity but poorer energy resolution permits a study of the continuum up to $\sim 20$ $\mathrm{keV}$. The imaging telescopes have yielded both low-resolution, spatially resolved spectral data (position sensitive detector = PSD) and higher resolution images with various transmission filters. The PSD allows us an observation of the spectral region containing the Fe- $\mathrm{L}$ complex as well as $\mathrm{Mg}$ and $\mathrm{Si}$ lines. A combination of these experiments gives an integral spectrum over the range $0.5-\sim 20 \mathrm{keV}$.

\section{OBSERVATIONAL RESULTS}

All instruments on board the EXOSAT satellite have been used for the observation of Cas A. The respective observation times are compiled in Table 1 . The instruments themselves have been described elsewhere (Taylor et al. 1981; de Korte et al. 1981; Peacock et al. 1981). Here we only summarize the most salient features (see Table 1).

The in-flight energy calibrations of the various instruments is briefly described below. The PSD was calibrated using a radioactive source with several emission lines (i.e., F-K $\alpha$ and $\mathrm{Al}-\mathrm{K} \alpha$ ) and was calibrated to an accuracy of about $20 \mathrm{eV}$. The calibration of the ME detectors was performed by observations of the Crab Nebula and comparing the spectrum with canonical values, i.e., a power-law spectrum with normalization constant $C=9.5$ photons $\mathrm{cm}^{-2} \mathrm{~s}^{-1} \mathrm{keV}^{-1}$ and photon index -2.1 , using a hydrogen column density of $3.5 \times 10^{21}$ $\mathrm{cm}^{-2}$ (obtained by analyzing the LE filter ratios). The accuracy of the energy calibration can be demonstrated to be better than $1 \%$.

We used observations of the Crab Nebula spectrum as well as lines from an internal $\mathrm{Pb}$ source to calibrate the GSPC to high accuracy $(\sim 25 \mathrm{eV}$ at $6 \mathrm{keV})$ and also took great care to remove all possible sources of systematic uncertainty (cf. Lamb et al. 1987).

Model spectra were fitted to the data using the $\chi^{2}$ minimization technique. The $\chi^{2}$ search was performed by conventional parameter adjustment after rebinning the data in bins of a width equal to one-third of the energy resolution, to insure that no information is lost and that one does not get an artificially reduced $\chi^{2}$ by oversampling (Davelaar 1979), and folding the model spectrum through the detector response matrix. For the calculation of CIE spectra the Mewe, Gronenschild, and van den Oord (1985) code was used. The NIE spectra were taken from Hamilton, Sarazin, and Chevalier (1983, HSC hereafter). The interstellar absorption data of Morrison and McCammon (1983) were used.

\section{a) Integral Spectrum}

Special care has been taken in the analysis of the low-energy data, $0.5-2.5 \mathrm{keV}$, since a strong correlation is expected between several spectral parameters. In particular the strength of the Fe-L line complex between 0.8 and $1.2 \mathrm{keV}$, which is of course a function of $\mathrm{Fe}$ abundance and plasma temperature, might be strongly anticorrelated with the spectral cutoff caused by interstellar absorption. Therefore the interstellar column density has been determined independently in order to have a consistency check on the value derived from the X-ray data. Thus, spatially resolved $\left(\sim 1^{\prime}\right) 21 \mathrm{~cm}$ radio continuum measurements were used to synthesize an average hydrogen column density of $1.47 \times 10^{22} \mathrm{~cm}^{-2}$ toward the remnant (Jansen et al. [1985] and references therein).

The $N_{\mathrm{H}}$ value derived from the X-ray data turns out to be $(1.38 \pm 0.09) \times 10^{22} \mathrm{~cm}^{-2}$, consistent with the radio data. This gives us confidence that the determination of the Fe- $\mathrm{L}$ flux is not impaired by its anticorrelation with the interstellar absorption.

To characterize the spectral parameters, the PSD and GSPC data were fitted to two simple models: a two-temperature plasma in CIE with variable abundances, which will be dealt

TABLE 1

EXOSAT DeteCtor Characteristics

\begin{tabular}{|c|c|c|c|}
\hline \multirow[b]{2}{*}{ DETECTOR } & \multirow{2}{*}{$\begin{array}{l}\text { ObseRvation TIME } \\
\text { (s) }\end{array}$} & \multicolumn{2}{|c|}{ RESOLUTION $^{\mathrm{a}}$} \\
\hline & & Spartial & Spectral \\
\hline CMA ........... & $10^{5}$ & $18^{\prime \prime}$ & (Broad-band filters) \\
\hline PSD ............ & $4 \times 10^{4}$ & $\begin{array}{l}100^{\prime \prime} \text { at } 0.8 \mathrm{keV} \\
60^{\prime \prime} \text { at } 2.0 \mathrm{keV}\end{array}$ & $44 \%$ at $1 \mathrm{keV}$ \\
\hline $\operatorname{Me} \operatorname{Ar} \ldots \ldots \ldots$ & $3 \times 10^{4}$ & $45^{\prime}$ collimator & $20 \%$ at $6 \mathrm{keV}$ \\
\hline $\begin{array}{l}\operatorname{Me~Xe~} \ldots \ldots \ldots \ldots \\
\text { GSPC } \ldots \ldots \ldots \ldots\end{array}$ & $\begin{array}{l}3 \times 10^{4} \\
10^{5}\end{array}$ & $45^{\prime}$ collimator & $\begin{array}{c}19 \% \text { at } 22 \mathrm{keV} \\
11 \% \text { at } 6 \mathrm{keV}\end{array}$ \\
\hline
\end{tabular}

${ }^{\text {a }}$ Given is the full width at half-maximum (FWHM). 

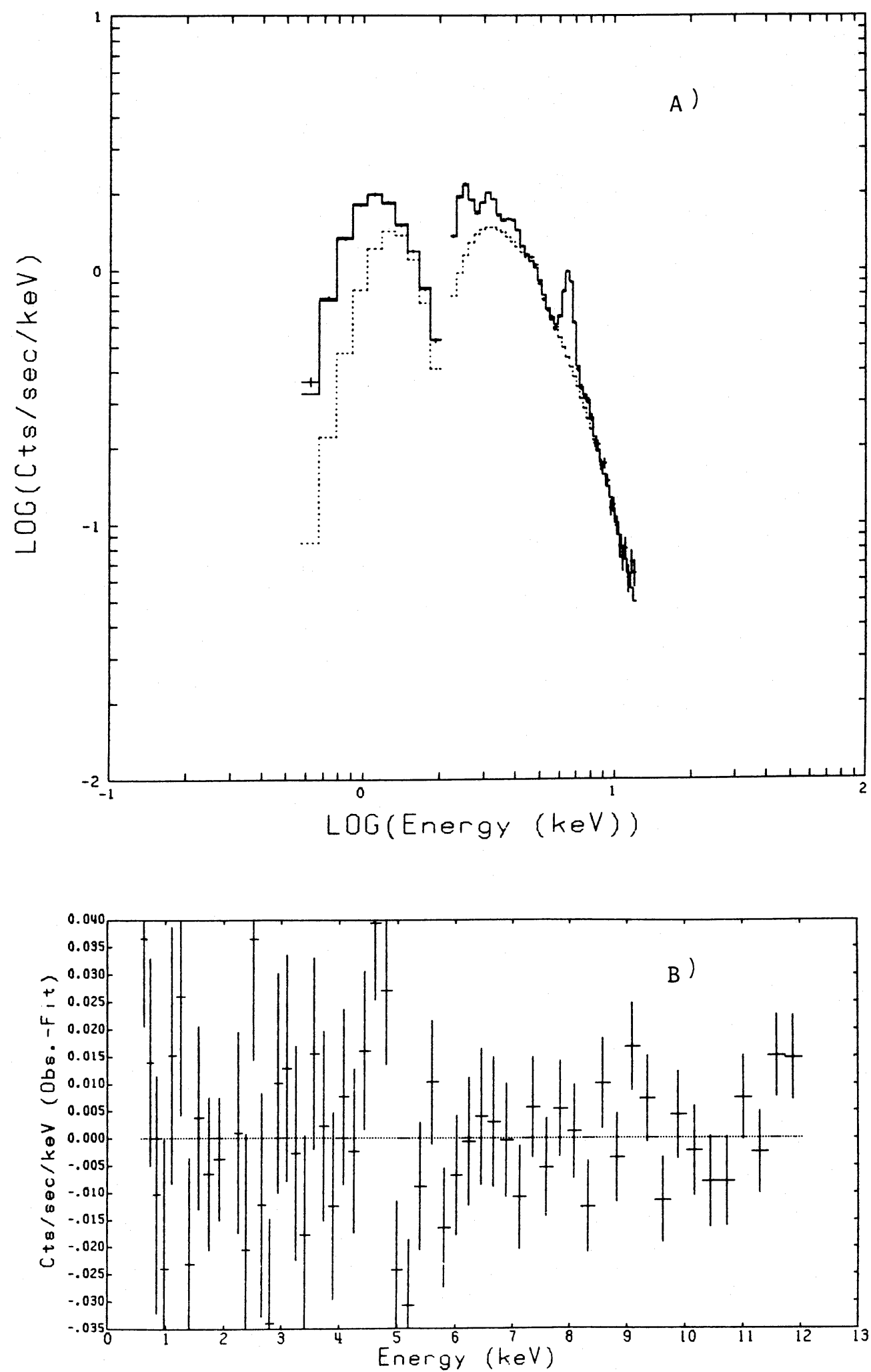

Fig. 1.- (a) The PSD and GSPC X-ray spectra for Cas A. The crosses show the pulse height spectra. The solid line represents the best-fit model spectrum (See also Table 2). The dashed line represents the sum of the low- and high-temperature bremsstrahlung continua. (b) Shown are the fitted residuals. 
TABLE 2

Results of SPECTRAL Fits to PSD and GSPC Data

\begin{tabular}{|c|c|c|c|c|}
\hline Element & $\begin{array}{l}\text { Energy } \\
(\mathrm{keV})\end{array}$ & $\begin{array}{l}\mathrm{EW}^{\mathrm{a}} \\
(\mathrm{eV})\end{array}$ & $\begin{array}{c}\text { Line Intensity } \\
\text { (photons } \mathrm{cm}^{-2} \mathrm{~s}^{-1} \text { ) }\end{array}$ & $\begin{array}{c}\text { CIE Temperature } \\
(\mathrm{keV})\end{array}$ \\
\hline 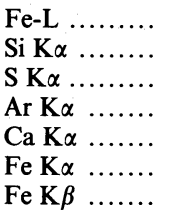 & $\begin{array}{l}0.77 \pm 0.04 \\
1.90 \pm 0.03 \\
2.46 \pm 0.016 \\
3.12 \pm 0.016 \\
3.88 \pm 0.023 \\
6.62 \pm 0.027 \\
7.84 \pm 0.06\end{array}$ & $\begin{array}{l}1450 \pm 180 \\
211 \pm 40 \\
446 \pm 17 \\
181 \pm 6 \\
124 \pm 6 \\
916 \pm 16 \\
123 \pm 20\end{array}$ & $\begin{aligned} 7.71 & \pm 1.19 \\
0.071 & \pm 0.010 \\
0.0572 & \pm 0.0017 \\
0.00957 & \pm 0.00033 \\
0.00316 & \pm 0.00015 \\
0.00446 & \pm 0.00007 \\
0.00036 & \pm 0.00005\end{aligned}$ & $\begin{array}{l}0.3-1.2 \\
1.1-1.25 \\
0.5-1.2 \\
2.0-2.3 \\
0.85-1.00 \\
0.68-0.79\end{array}$ \\
\hline \multicolumn{5}{|c|}{ 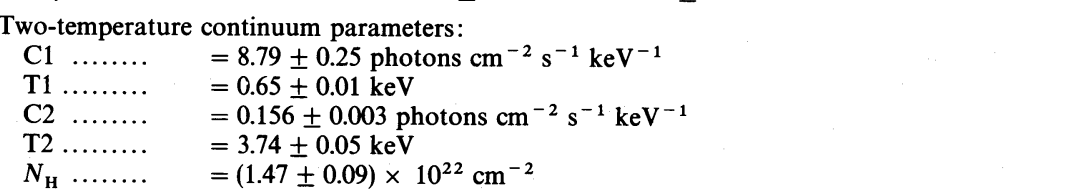 } \\
\hline
\end{tabular}

a Equivalent width of the line with respect to the two underlying continua.

b These are the temperature ranges needed to explain the observed energies if the emission were to originate from a plasma in CIE.

with in the discussion section, and a two-temperature thermal bremsstrahlung (TB) model plus emission lines. For these fits the ME data have not been used, since its relatively poor energy resolution would cause blurring out of the line features as observed by the GSPC. Fits with a single temperature bremsstrahlung model plus emission lines were found to be unacceptable. The two-temperature TB model was used in conjunction with seven Gaussian emission lines to model the $\mathrm{K} \alpha$ lines of $\mathrm{Si}, \mathrm{S}, \mathrm{Ar}, \mathrm{Ca}$, and $\mathrm{Fe}$ as well as the $\mathrm{Fe}-\mathrm{L}$ and $\mathrm{Fe}-\mathrm{K} \beta$ lines. In fitting this model the normalization constants, continuum temperatures, line intensities, line centroids, and some line widths were left free to vary. Such a model is adequate to parameterize the data in terms of continuum temperatures, line centroids, and equivalent widths, which can subsequently be compared to model predictions. The resultant fit and the residuals are shown in Figure 1 and the fit parameters are given in Table 2. When compared to the Tenma results (Tsunemi et al. 1986) the line centroids as well as the continuum intensity are compatible, whereas our line intensities are systematically higher $(\sim 25 \%)$, which might be due to the exact detector parameters (e.g., spectral resolution) implemented in the spectral fit procedure.

Care should be taken in directly comparing the line intensities and equivalent widths to model predictions because they are given with respect to a two-temperature continuum model.

In order to study the possible existence of a third, hightemperature, component as was reported by HEAO A2 (Pravdo and Smith 1979) the ME data in the range 4-20 keV has been carefully analyzed. The ME data were fitted to a simple two-component thermal bremsstrahlung spectrum with a single emission line corresponding to $\mathrm{Fe}-\mathrm{K}$. The luminosity of the very high temperature component was fixed to the value inferred from the $H E A O$ data. The fit results are given in Table 3 , and the best fit is shown in Figure 2. From this table it can be seen that the best fit has no very hard component and a lowtemperature value of $3.96 \mathrm{keV}$. The fit is noticeably poorer when the low temperature is fixed at the value found for the PSD + GSPC (3.76 keV), which might suggest some harder emission but of a much reduced strength as compared to the $H E A O$ result.

Thus, our data do not require the presence of a very high temperature component, and even excludes a component of the luminosity inferred from the $H E A O$ data unless its temperature is in excess of $25-30 \mathrm{keV}$ (see Table 3).

\section{b) Spectral Morphology}

The high-resolution image enables us to confirm the remnant's morphology, which has also been studied by Fabian et al. (1980). The X-ray image reveals a clump, ringlike structure, as well as a weak outer shell of emission, all of which have also been seen by the HRI. The outer shell of emission coincides with the maximum in the radio contours (see Fig. 3 [pl. 10]). The position angle (0-360 degrees) averaged radii for these components, as well as their errors, are given in Table 4 together with the values derived by Fabian et al. (1980). The radii for the clump, ringlike structure, both for the EXOSAT CMA and the Einstein HRI, were derived from the X-ray image which was deconvolved to remove the effects of spatial blurring by the instrument point response function. The radii for the shock emission were derived by comparing the deconvolved data with a uniform spherical shell model, projected onto a plane perpendicular to the line of sight.

The third, halo component which was discovered with Einstein by Steward, Fabian, and Seward (1981) is also present in

TABLE 3

\begin{tabular}{|c|c|c|c|}
\hline \multicolumn{2}{|c|}{$\begin{array}{l}\text { ContinuUum } \\
(\mathrm{keV})\end{array}$} & \multirow[b]{2}{*}{$\chi^{2}$} & \multirow[b]{2}{*}{ d.o.f } \\
\hline Low Temp. & High Temp. & & \\
\hline $3.76^{\mathrm{a}} \ldots \ldots \ldots$ & $\ldots$ & 43.2 & 19 \\
\hline $3.96 \ldots \ldots \ldots$ & & 35.0 & 18 \\
\hline $3.76^{\mathrm{a}}$. & 20 & 82.0 & 19 \\
\hline $3.32 \ldots \ldots \ldots$ & 20 & 44.1 & 18 \\
\hline $3.76^{\mathrm{a}} \ldots \ldots \ldots$ & 25 & 74.4 & 19 \\
\hline $3.40 \ldots \ldots \ldots$ & 25 & 41.1 & 18 \\
\hline $3.76^{\mathrm{a}} \ldots \ldots \ldots$ & 30 & 55.6 & 19 \\
\hline $3.48 \ldots \ldots \ldots$ & 30 & 41.1 & 18 \\
\hline $3.76^{\mathrm{a}} \ldots \ldots \ldots$ & 40 & 47.9 & 19 \\
\hline $3.54 \ldots \ldots \ldots$ & 40 & 40.4 & 18 \\
\hline
\end{tabular}

${ }^{a}$ In this case the low temperature value was fixed, at the best-fit value found for the PSD and GSPC data. 


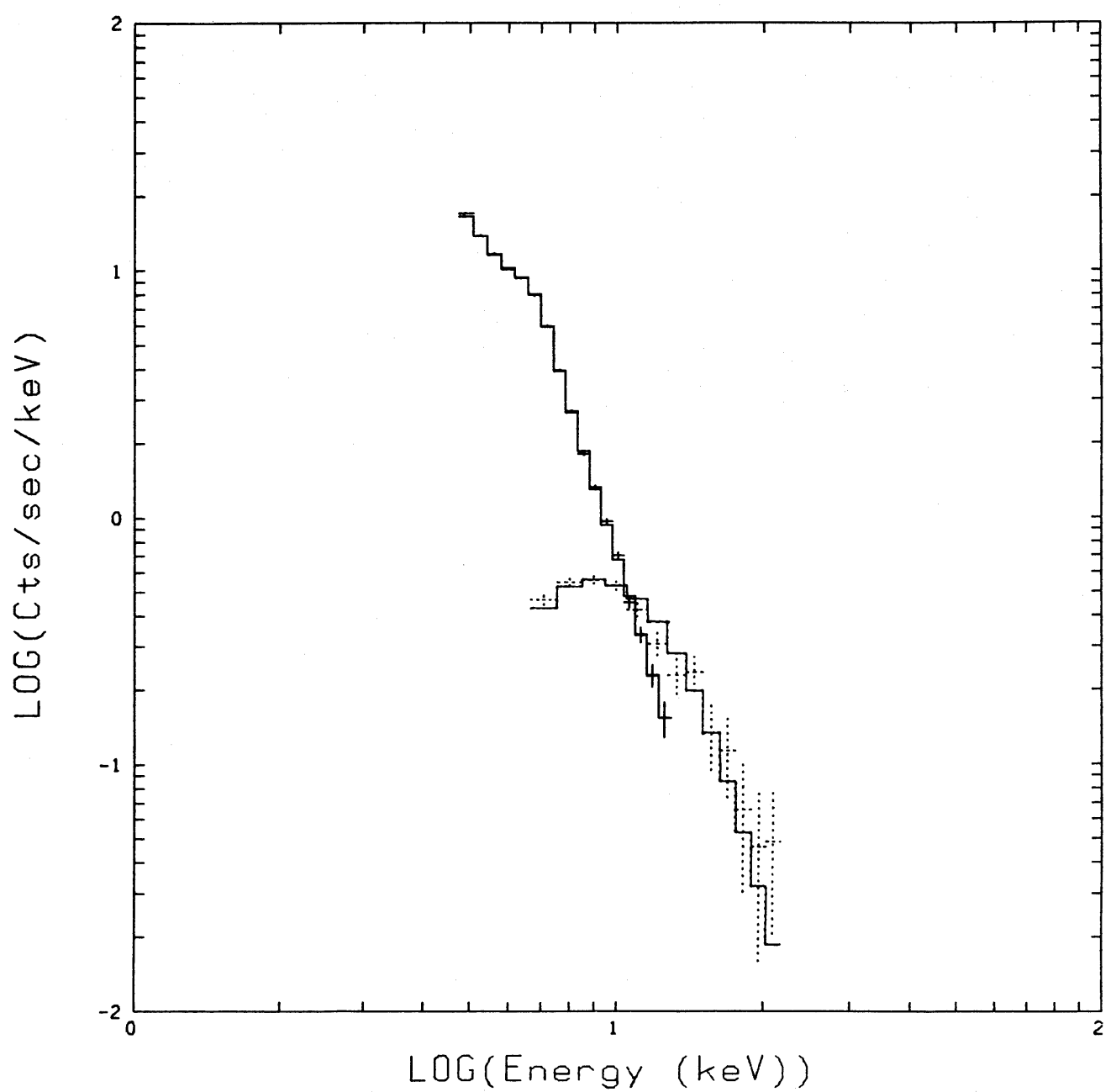

Fig. 2.-The ME Ar and Xe X-ray spectra for Cas A. The solid crosses show the Ar pulse height spectra, the dashed crosses show the Xe pulse height spectra. The solid lines show the best-fit ME model spectra as given in Table 3, folded through the ME Ar and Xe detector response matrix, respectively.

our data as is shown in Figure 4. Outside the shock radius $\left(145^{\prime \prime}\right)$ a significant flux is still measured up to about $300^{\prime \prime}$ radius. This component contains about $10 \%$ of the total X-ray flux in the CMA X-ray image. When one interprets this halo as being caused by the scattering of X-rays on interstellar dust, we can use the data to derive some dust parameters by making use of the formula given by Mauche and Gorenstein (1986). An average grain size of $0.17-0.18 \mu \mathrm{m}$ and a linear absorption coefficient between 0.028 and $0.041 \mathrm{kpc}^{-1}$ is derived under the assumption that the average energy of the halo in the EXOSAT passband is $1 \mathrm{keV}$, and the distance to Cas A equals $2.92 \mathrm{kpc}$ (Braun 1985). These results are consistent with those obtained by Mauche and Gorenstein (1986) for other galactic

TABLE 4

Radil of SeVeral Components

\begin{tabular}{|c|c|c|}
\hline Description & $\begin{array}{c}\text { CMA } \\
\text { Deconvolved }\end{array}$ & $\begin{array}{c}\text { HRI } \\
\text { Deconvolved }\end{array}$ \\
\hline Average radius clumpy ring & $92^{\prime \prime} \pm 10^{\prime \prime}$ & $102^{\prime \prime} \pm 8^{\prime \prime}$ \\
\hline Inner radius clumpy ring ... & $71^{\prime \prime} \pm 10^{\prime \prime}$ & $90^{\prime \prime} \pm 8^{\prime \prime}$ \\
\hline Outer radius clumpy ring ............. & $126^{\prime \prime} \pm 10^{\prime \prime}$ & $124^{\prime \prime} \pm 8^{\prime \prime}$ \\
\hline Average shock radius ................ & $\sim 145^{\prime \prime}-20$ & $\sim 160^{\prime \prime}-$ \\
\hline Shock thickness .................... & $\sim 25^{\prime \prime}$ & $\sim 20^{\prime \prime}$ \\
\hline
\end{tabular}

sources. The supernova remnant can thus be assumed to consist of two components, a clumpy ring and a weak outer shell, while the third component, a scattering halo, is not physically related to the material heated by the blast wave.

A two-component structure can also be observed in the spectral analysis of radial segments in the PSD data. This analysis was carried out by convolving the images in nine spectral bands to equal spatial resolution (i.e., a Gaussian of 100" FWHM), determining the counts in radial annuli, and performing spectral fits with simple-temperature CIE spectra assuming cosmic abundances for all elements but silicon. It should be noted that the derived temperature is not so much of direct physical relevance but is indicative of a change in spectral shape. The variation of the derived CIE temperature with radius, summed over all position angles, is shown in Figure 5. Going outward the data show a significant increase of the radial temperature, which has a broad maximum in the range $R=160^{\prime \prime}-190^{\prime \prime}$. Further outward the statistical data quality does not allow for an accurate determination of the temperature. The radial position and the value of this maximum in the temperature profile is consistent with the identification of the weak, extended emission plateau with the hightemperature component $(3.7 \mathrm{keV}$ continuum) observed with the GSPC and ME and the low-temperature component with 


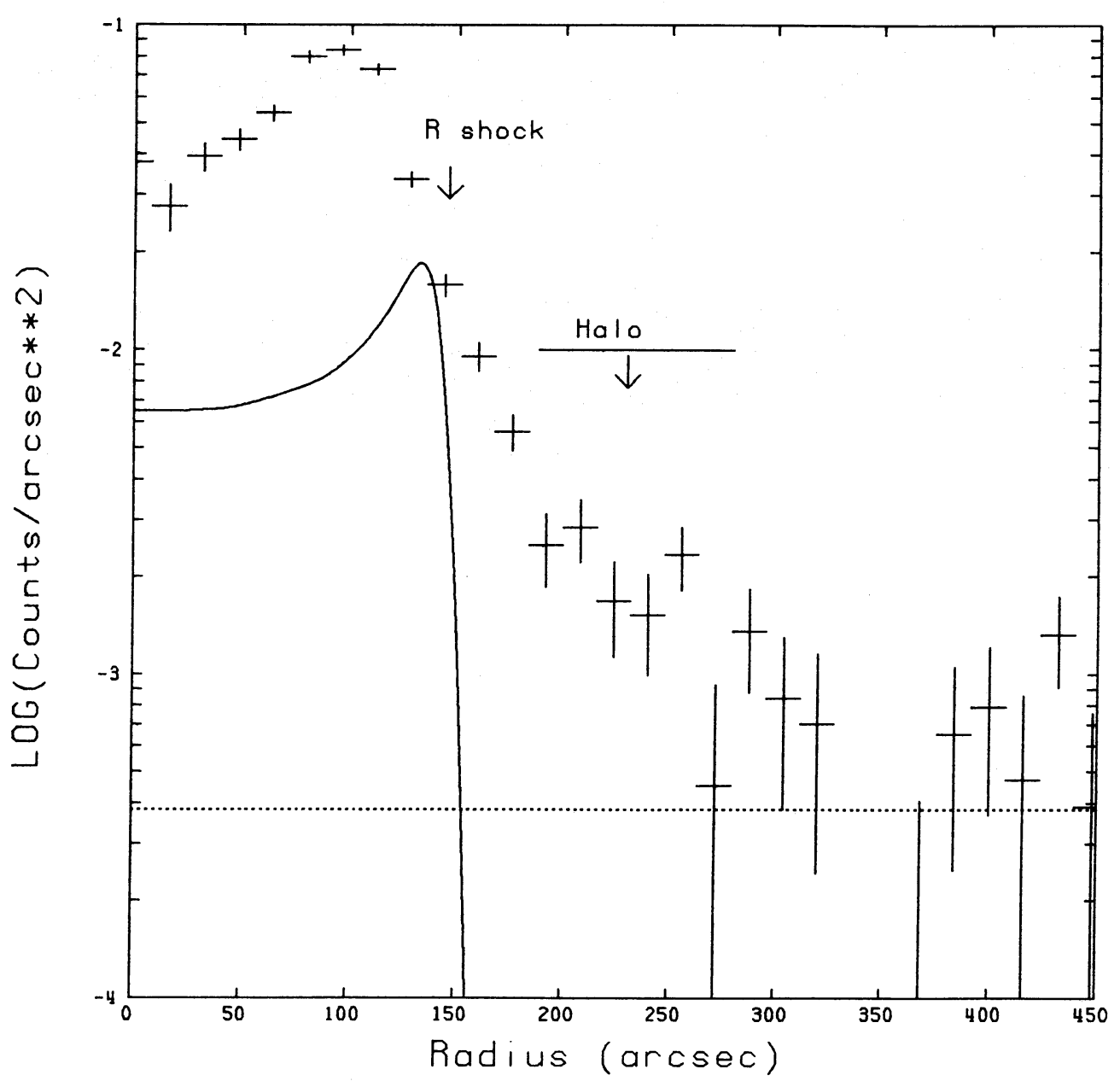

FIG. 4.-Shown as crosses is the azimuthally integrated radial brightness profile of the CMA image. The dashed line represents a residual detector background level, while the solid line represents the radial brightness profile as expected from a pure Sedov remnant with a shock radius as indicated. This shock radius is derived from other data (see Table 4), the solid line was normalized to have the same luminosity per surface area, for this shock emission, as in Fig. 5. It is obvious that there is excess radiation present outside $R \sim 180^{\prime \prime}$, which can be explained by a scattering halo.

the clumpy ring like structure. This can be demonstrated as follows.

In the PSD pass band, the relative contribution of the hightemperature component vis-à-vis the low-temperature component increases when going to larger radii, resulting in a gradual hardening of the resulting spectral distribution. Since the absolute contribution of the high-temperature component to the total flux in the PSD passband remains minor, the net increase in apparent temperature amounts to only $0.05 \mathrm{keV}$ when summed over all position angles. Comparison of the other radial temperature plots in Figure 5 shows a maximum temperature excursion for position angles between $200^{\circ}$ and $280^{\circ}$, where the emission from the clumpy component is lowest. The spatial coincidence of the temperature maximum with the weak outer shell, verified by folding of the high- and lowtemperature component with the PSD spatial resolution and predicting both position and value of this maximum (see Fig. 5), supports the identification of this shell with the hightemperature component and thus the interaction site of the blast wave with the ISM. The azimuthal variation of the radial temperature excursion can thus be entirely ascribed to the variation of the emission from the inner, clumpy, component.
The data are consistent with a uniformly emitting shell of $\mathrm{X}$-ray emitting gas with outer radius of $145^{\prime \prime}$ and thickness $\sim 25^{\prime \prime}$. In the following discussion we shall assume these values to describe the outer, high-temperature component.

\section{DISCUSSION}

From the observed emission-line energies one can derive the corresponding temperatures needed for a plasma in CIE to produce such a line. The temperatures derived in this way for the various lines are given in Table 2.

Although some of the temperature ranges overlap we know from the $\mathrm{Fe}-\mathrm{K} \alpha$ line alone that in order to produce the observed line energy of $6.62 \pm 0.3 \mathrm{keV}$ from a plasma in CIE, one would require a plasma temperature between 0.85 and 1.0 $\mathrm{keV}$. To produce the observed line strength from such a plasma, however, would require an $\mathrm{Fe}$ overabundance of more than 14 relative to solar. Such an overabundance of $\mathrm{Fe}$, however, is ruled out since it would produce Fe-L emission far in excess of what has been observed. Moreover, the energy centroid of the Fe-K $\alpha$ line cannot be explained by Doppler shift of the line from a plasma in CIE, since integration over a 

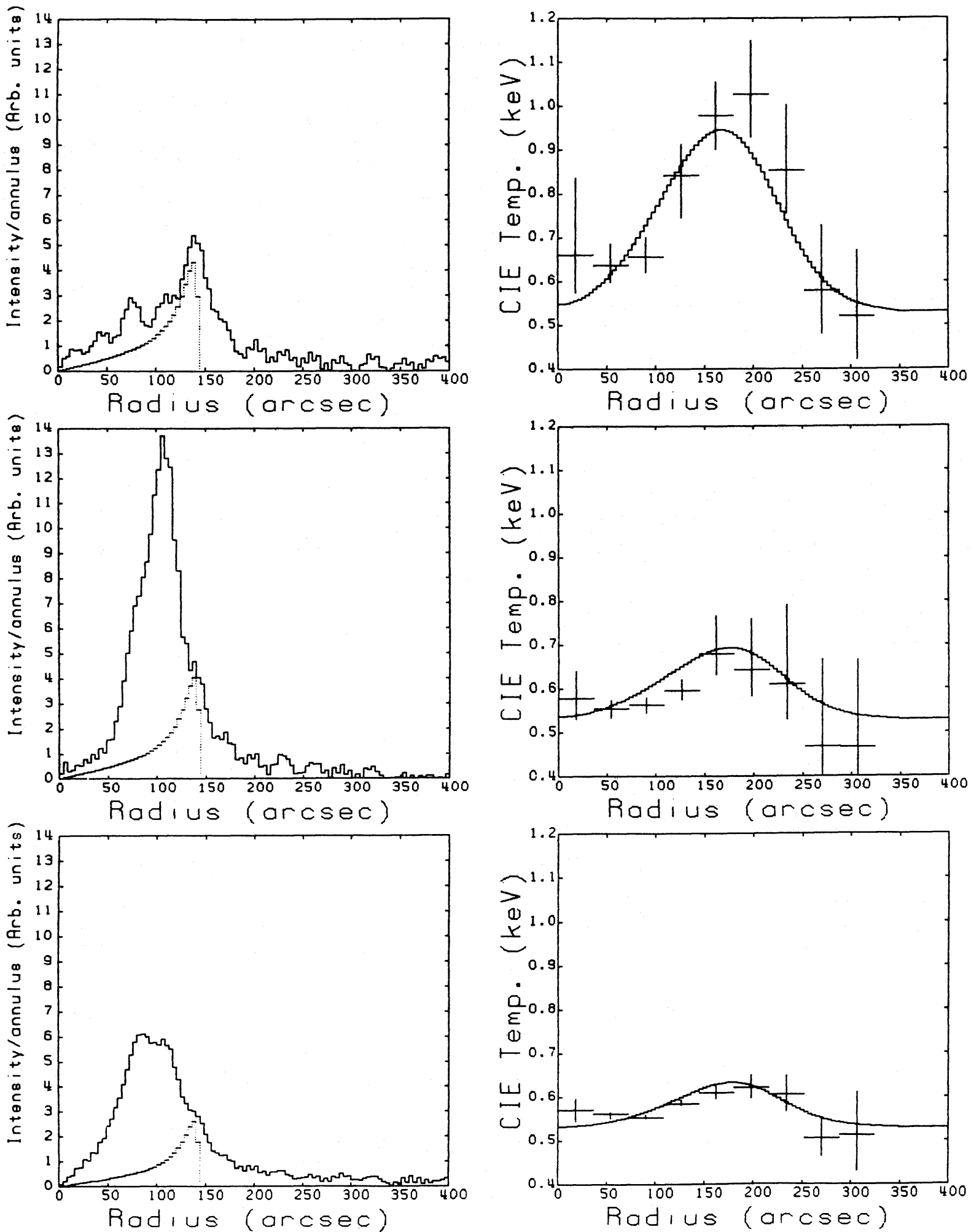

FIG. 5.-The CMA radial brightness profile is shown as a solid line in the three panels in the left-hand column for position angles (from top to bottom); $200<\mathrm{pa}<280,72<\mathrm{pa}<147$ and $0<\mathrm{pa}<360$, respectively. The dashed lines represent the profiles expected for a Sedov remnant. The crosses in the three panels in the right-hand column represent the radial temperature profile as measured with the PSD for the same respective sectors. The solid line is a temperature profile predicted by identifying the shock emission in the CMA brightness profiles (i.e., the dashed line) with the $3.7 \mathrm{keV}$ temperature component, convolving this with the PSD spatial resolution, and thus predict the PSD temperature profile by translating the radial percentage contribution of this component in an apparent temperature rise of the PSD spectrum. 
more or less spherically expanding remnant results predominantly in line broadening rather than line shift. ${ }^{2}$

We conclude, therefore, that the Cas A X-ray spectrum cannot be fitted by a two-temperature plasma in CIE. A twotemperature model was consistent with the Einstein SSS results. We can exclude such a model because the broader EXOSAT energy range provides additional information (principally the energy and intensity of the Fe-K lines) which was not available to the SSS.

In order to derive some parameters of the SNR, we have used the Sedov solution for the hydrodynamics of a blast wave (Sedov 1959). As we have identified the high-temperature (3.74 $\mathrm{keV}$ ) component with the emission from the blast wave; and also because Hamilton and Sarazin (1984) have shown that the NIE spectral shape of a Sedov remnant is representative of several other types of SNR, we have used the NIE spectra from HSC to describe this component.

We have assumed the electrons and ions to be in thermal equilibrium $\left(T_{e}=T_{i}\right)$. The reasons for this assumption are the following.

1. In order to produce the observed $\mathrm{Fe}-\mathrm{K} \alpha$ energy of 6.62 $\mathrm{keV}$, as well as a hard X-ray spectrum with $T_{e} \sim 3.74 \mathrm{keV}$ (as measured) from a plasma in NIE where $T_{e} \neq T_{i}$ values for $\eta$ in excess of even $10^{54}-10^{55}$ ergs $\mathrm{cm}^{-6}$ are required (see HSC, eq. [8] as well as extrapolating their Fig. 8). Such a value for $\eta$ is untenable, since it would produce an X-ray luminosity for Cas A far in excess of what is measured, even for a filling factor as low as 0.1 .

2. For $T_{e} \neq T_{i}$ it is assumed that the shock interacts with the electrons and ions as if it were two decoupled fluids which are heated afterward by Coulomb processes. More detailed models, however, take charge coupling as well as other collective plasma effects into account (e.g., viscosity and heat conduction; see Mihalas and Mihalas 1984) which results in an $T_{i} / T_{e}$ ratio after the shock ranging from 1 to 5 instead of 1800 for the case of the two-fluid approximation.

Taking then that $T_{e}=T_{i}$ and that the shock temperature $T_{s}=T_{e} / 1.3$, where $T_{e}$ equals the value measured for the high $T$ component, i.e., $3.74 \mathrm{keV}$, we will use the HSC model for further interpretation of the blast-wave component. This model is characterized by two parameters; $\eta\left(=n_{0}{ }^{2} E_{0}, n_{0}\right.$ is the ambient interstellar density and $E_{0}$ the initial explosion energy) and the shock temperature $T_{s}$. Important parameters can be obtained by using the Sedov equations as given by HSC:

$$
\begin{aligned}
t & =4015 \eta_{51}{ }^{-1 / 6} T_{s 7}-5 / 6 \sqrt{E_{51}} \mathrm{yr} \\
r_{s} & =8.62 \eta_{51}{ }^{-1 / 6} T_{s 7}{ }^{-1 / 3} \sqrt{E_{51}} \mathrm{pc} .
\end{aligned}
$$

From which follows

$$
r_{s}=2.147 \times 10^{-3} \sqrt{T_{s 7}} t \mathrm{pc}
$$

where $r_{s}$ is the shock radius, $T_{s 7}$ is the shock temperature in units $10^{7} \mathrm{~K}, E_{51}$ is the explosion energy in units $10^{51}$ ergs. $\eta_{51}=n_{0}^{2} E_{51}$ ergs cm${ }^{-6}$, and $t$ is the age in years. These equations can be solved since we know that $r_{s}=145^{\prime \prime}$, the distance to Cas $\mathrm{A}$ is $2.92 \pm 0.1 \mathrm{kpc}$ (Braun 1985) and that $T_{s 7}=3.34$. Therefore, $r_{s}=2.05 \pm 0.14 \mathrm{pc} ; t=522 \pm 36 \mathrm{yr}$. We can now

\footnotetext{
2 Taking the uncertainties in the detector resolution into account we can use the observed $\mathrm{Fe}-\mathrm{K} \alpha$ line width to derive an upper limit on the expansion velocity for a spherically symmetric model. The derived upper limit is $\sim 2500$ $\mathrm{km} \mathrm{s}^{-1}$, roughly in agreement with the optically observed velocities of the fast moving knots as well as the expansion velocity of more than $2000 \mathrm{~km} \mathrm{~s}^{-1}$ determined by the FPCS (Markert et al. 1983).
}

calculate an estimate for the ambient density $n_{0}$, independent from the Sedov equations (and thus also for the swept-up $M_{s}$, since $M_{s}=\mu n_{0} V$; with $\mu$ the average molecular weight and $V$ the remnant volume) by using the measured luminosity of the high-temperature bremsstrahlung component $\left(L_{x}=1.78\right.$ $\times 10^{36}$ ergs s $^{-1}$ ) to calculate $N_{e}$ (the electron density in the emitting region) from

$$
\frac{d P_{B}}{d V}=7.6 \times 10^{-24} \sqrt{T_{7}} N_{e}^{2} \operatorname{ergs~} \mathrm{cm}^{-3} \mathrm{~s}^{-1}
$$

(Zombeck [1982]; $P_{B}=$ power emitted in bremsstrahlung radiation). For the emitting volume we take the volume associated with the blast wave (effectively a spherical shell with thickness $r_{s} / 12$ and outer radius $r_{s}$ ) and get $N_{e}=22.9 \pm 4.8 \mathrm{~cm}^{-3}$ and thus $n_{0}=5.7 \pm 1.2 \mathrm{~cm}^{-3}$ since for a strong shock $N_{e}=$ $4 n_{0}$. From these data we derive the swept-up mass $M_{s}$ to be $5.8 \pm 1.2 M_{\odot}$ and use equation (2) to derive $E_{51}=0.26 \pm 0.06$.

The value obtained for $\eta$ is $\left(8.4_{-4}^{+7}\right) \times 10^{51}$ ergs $\mathrm{cm}^{-6}$ which is consistent with the value obtained by matching the predicted and the measured line-energy centroids for the $\mathrm{Fe}-\mathrm{K} \alpha$ and $\mathrm{Ca}-\mathrm{K} \alpha$ lines (Fig. 8 of HSC). The expected and observed line strengths as well as their ratios, for the NIE model with this value of $\eta$, are given in Table 5 . These ratios cannot directly be translated into abundances since the observed line strengths were derived using a two-temperature continuum. The high ratios obtained at low energies are clearly indicative of the presence of a low-temperature component, which might well be associated with the reverse shocked ejecta, while the high-temperature component can be ascribed to emission from material in the interstellar medium (ISM) which was shocked by the primary blast wave. A second component is required because an overabundance of approximately 15 is needed to explain the Fe-L emission in a one-component NIE spectrum, while the abundance derived from the $\mathrm{Fe}-\mathrm{K} \alpha$ line is about solar. This scenario is further supported by the observations in the PSD (see Fig. 5) where the interior is cool and the temperature is highest at the point where the blast wave is expected to interact with the ISM, i.e. $R \sim 145^{\prime \prime}$.

The observed excess Fe-L emission, which is not explained by the one-temperature NIE model, can in principle be used to estimate the amount of currently emitting stellar ejecta and thus the mass of the progenitor star. The simplest and most straightforward approach would be to assume a plasma in CIE to be the emitter of the excess Fe-L emission. This leads to an estimate, albeit systematically uncertain, for the currently emitting stellar ejecta of $\sim 15 M_{\odot}$.

In order to obtain an NIE estimate for the ejected mass, one would need to produce NIE spectra for a reverse shock from the HSC data. This method is described by Hamilton and Sarazin (1984) for self-similar solutions of the interaction of

TABLE 5

NIE ANALYSIS Results

\begin{tabular}{cccr}
\hline \hline Element & $\begin{array}{c}\text { Observed Intensity } \\
\left.\text { (photons } \mathrm{cm}^{-2} \mathrm{~s}^{-1}\right)\end{array}$ & $\begin{array}{c}\text { Predicted NIE Intensity } \\
\left(\text { photons } \mathrm{cm}^{-2} \mathrm{~s}^{-1}\right)\end{array}$ & Ratio \\
\hline $\mathrm{Fe}-\mathrm{L} \ldots \ldots \ldots \ldots$ & 7.71 & 0.458 & 16.8 \\
$\mathrm{Si} \ldots \ldots \ldots \ldots \ldots$. & 0.071 & 0.023 & 3.1 \\
$\mathrm{~S} \ldots \ldots \ldots \ldots \ldots$. & 0.057 & 0.0091 & 6.3 \\
$\mathrm{Ar} \ldots \ldots \ldots \ldots \ldots$. & 0.0096 & No prediction & \\
$\mathrm{Ca} \ldots \ldots \ldots \ldots \ldots$ & 0.0032 & 0.00073 & 4.4 \\
$\mathrm{Fe}-\mathrm{K} \alpha \ldots \ldots \ldots \ldots$ & 0.0045 & 0.0023 & 2.0 \\
$\mathrm{Fe}-\mathrm{K} \beta \ldots \ldots \ldots \ldots$ & 0.00036 & No prediction & \\
\hline
\end{tabular}


stellar ejecta with an external medium, for the two models typical for a Type I $\left(\rho_{\mathrm{ej}} \propto \boldsymbol{r}^{-7} ; \rho_{\mathrm{ext}}=\right.$ constant $)$ and Type II supernova $\left(\rho_{\mathrm{ej}} \propto r^{-12} ; \rho_{\mathrm{ext}} \propto r^{-2}\right)$. Chevalier (1982) derived, for these two cases, a mass ratio of 0.50 and 2.7, respectively, We, however, do not have the possibility to test which of these two models produces an aceptable fit to the spectrum.

More importantly, every one of the beforementioned methods leads to a mass estimate which is hampered by systematic uncertainties (i.e., the highly uncertain amount of $\mathrm{Fe}$ produced in supernova explosions [Johnson and Yahil 1984]).

The analysis of the Tenma data (Tsunemi et al 1985) assumes that the total X-ray emission comes from shock-heated enriched ejecta. Such a model is adequate in their case, but for the extended spectral range and imaging capability of EXOSAT, a two-component NIE spectrum is required. The Tenma interpretation of the $3.74 \mathrm{keV}$ component as being due to stellar ejecta heated by the reverse shock is, almost certainly, incorrect, since it is clear from our observations that the higher temperature (primary shock) region is the $3.74 \mathrm{keV}$ component. We believe that this plasma is not composed of ejecta at all and is thus representative of the material surrounding the presupernova.

\section{CONCLUSION}

We have interpreted the X-ray emission from Cas A as originating from a high- and low-temperature plasma. The high- temperature plasma, for which we fitted a continuum temperature of $T_{s}=3.3 \times 10^{7} \mathrm{~K}$, is most probably circumstellar material which has been shocked by the primary blast wave and has not attained ionization equilibrium. The lowtemperature plasma (whose continuum temperature is fitted at $7.5 \times 10^{6} \mathrm{~K}$ ) is most likely reverse shocked ejecta, a hypothesis which is independently supported by the radial temperature distribution for several position angles of the emission in the $0.5-2.1 \mathrm{keV}$ band.

We have attempted to compute the density and mass of the $\mathrm{X}$-ray emitting material in both the ejecta and in the swept-up circumstellar material. The swept-up mass is determined from the observed luminosity of the high-temperature continuum, under the assumption of thermal bremsstrahlung emission. We find $M_{s}$ to be $5.8 \pm 1.2 M_{\odot}$. A rough estimate for the amount of currently emitting stellar ejecta can be derived as $15 M_{\odot}$; although since this estimate is hampered by systematic uncertainties it is intended only to indicate an order of magnitude.

The Laboratory for Space Research Leiden and the Laboratory for Space Research Utrecht are sponsored by the Netherlands Organisation for the Advancement of Pure Research (ZWO). We are grateful for valuable comments by an anonymous referee.
Arnett, W., 1975, Ap. J., 195, 727

Becker, R. H., Holt, S. S., Smith, B. W., White, N. E., Boldt, E. A., Mushotzky, R. F., and Serlemitsos, P. J. 1975, Ap. J.(Letters), 234, L73.

Braun, R. 1985, Ph.D. thesis, Leiden State University.

Charles, P. A., Culhane, J. L., Zarnecki, J. C., and Fabian, A. C. 1975, Ap. J., 197, L61.

Chevalier, R. A. 1982, Ap. J., 258, 790.

Chevalier, R. A., and Kirshner, R. P. 1978, Ap. J., 219, 931.

Davelaar, J. 1979, Ph.D. thesis, Leiden State University.

Davison, P. J. N., Culhane, J. L., and Mitchell, R. J. 1976, Ap. J. (Letters), 206, L37.

de Korte, P. A. J., et al. 1984, Space Sci. Rev., 30, 495

Fabian, A. C., Willingale, R., Pye, J. P., Murray, S. S., and Fabbiano, G. 1980, M.N.R.A.S., 193, 175 .

Hamilton, A. J. S., and Sarazin, C. L. 1984, Ap. J., 284, 601.

Hamilton, A. J. S., Sarazin, C. L., and Chevalier, R. A. 1983, Ap. J. Suppl., 51, 115 (HSC).

Itoh, H. 1977, Pub. Astr. Soc. Japan, 29, 813.

Itoh, H., and Fabian, A. C., 1984, M.N.R.A.S., 208, 645.

Jansen, F. A., et al. 1984, Adv. Space Res., 5, No. 3, 49.

Johnston, M. D., and Yahil, A. 1984, Ap. J., 285, 587.

Lamb, P., et al., 1987, to be published.

Markert, T. H., Canizares, C. R., Clark, C. W., and Winkler, P. F. 1983, Ap. J., 268, 134.

Mauche, C. W., and Gorenstein, P. 1986, Ap. J., 302, 371.

McKee, C. F. 1974, Ap.J., 188, 335.

\section{REFERENCES}

Mewe, R., and Gronenschild, E. H. B. M. 1985, Astr. Ap. Suppl., 62, 197.

Mihalas, D., and Mihalas, B. W. 1984, Foundations of Radiation Hydrodynamics (Oxford: Oxford University Press).

Morrison, R., and McCammon, D. 1983, Ap. J., 270, 119.

Murray, S. S., Fabbiano, G., Fabian, A. C., Epstein, A., and Giacconi, R. 1979, Ap. J. (Letters), 234, L69.

Peacock, A., Andresen, R. D., Manzo, G., Taylor, B. G., Villa, G., Re, S., Ives, J. C., and Kellock, S. 1981, Space Sci. Rev., 30, 525.

Pravdo, S. H., and Nugent, J. J. 1983, in IAU Symposium 101, Supernova remnants and their $X$-ray emission, ed. J. Danziger and P. Gorenstein (Dordrecht: Reidel), p. 29.

Pravdo, S. H., and Smith, B. W. 1979, Ap. J (Letters), 234, L195.

Sedov, L. 1959, Similarity Methods in Mechanics (New York: Academic).

Serlemitsos, P. J., Boldt, E. A., Holt, S. S., Ramaty, R., and Brisken, A. F. 1973, Ap. J. (Letters), 184, L1.

Stewart, G. C., Fabian, A. C., and Seward, F. D. 1983, in IAU Symposium 101, Supernova remnants and their X-ray Emission, ed. J. Danziger and P. Gorenstein (Dordrecht: Reidel), p. 59.

Taylor, B. G., Andresen, R. D., Peacock, A., and Zobl, R. 1981, Space Sci. Rev., 30, 479 .

Tsunemi, H., Yamashita, K., Masai, K., Hayakawa, S., and Koyama, K. 1986, Ap. J., 306, 248.

Tuffs, R. J. 1986, M.N.R.A.S., 219, 13

van den Bergh, S., and Kamper, K. W. 1983, Ap. J., 268, 129

Zombeck, M. V. 1982, Handbook of Space Astronomy and Astrophysics (Cambridge: Cambridge University Press), p. 200.

\section{J. A. M. BleEKER: Laboratory for Space Research Utrecht, Beneluxlaan 21, 3527 HS Utrecht, The Netherlands}

P. A. J. DE KORTE, and F. JANSEN: Laboratory for Space Research Leiden, Niels Bohrweg 2, Leiden, The Netherlands

A. Peacock and A. Smith: Space Science Department of ESA, ESTEC, Postbus 299, 2200 AG Noordwijk, The Netherlands

N. E. White: EXOSAT Observatory, Space Science Department of ESA, ESTEC, Postbus 299, 2200 AG Noordwijk, The Netherlands 

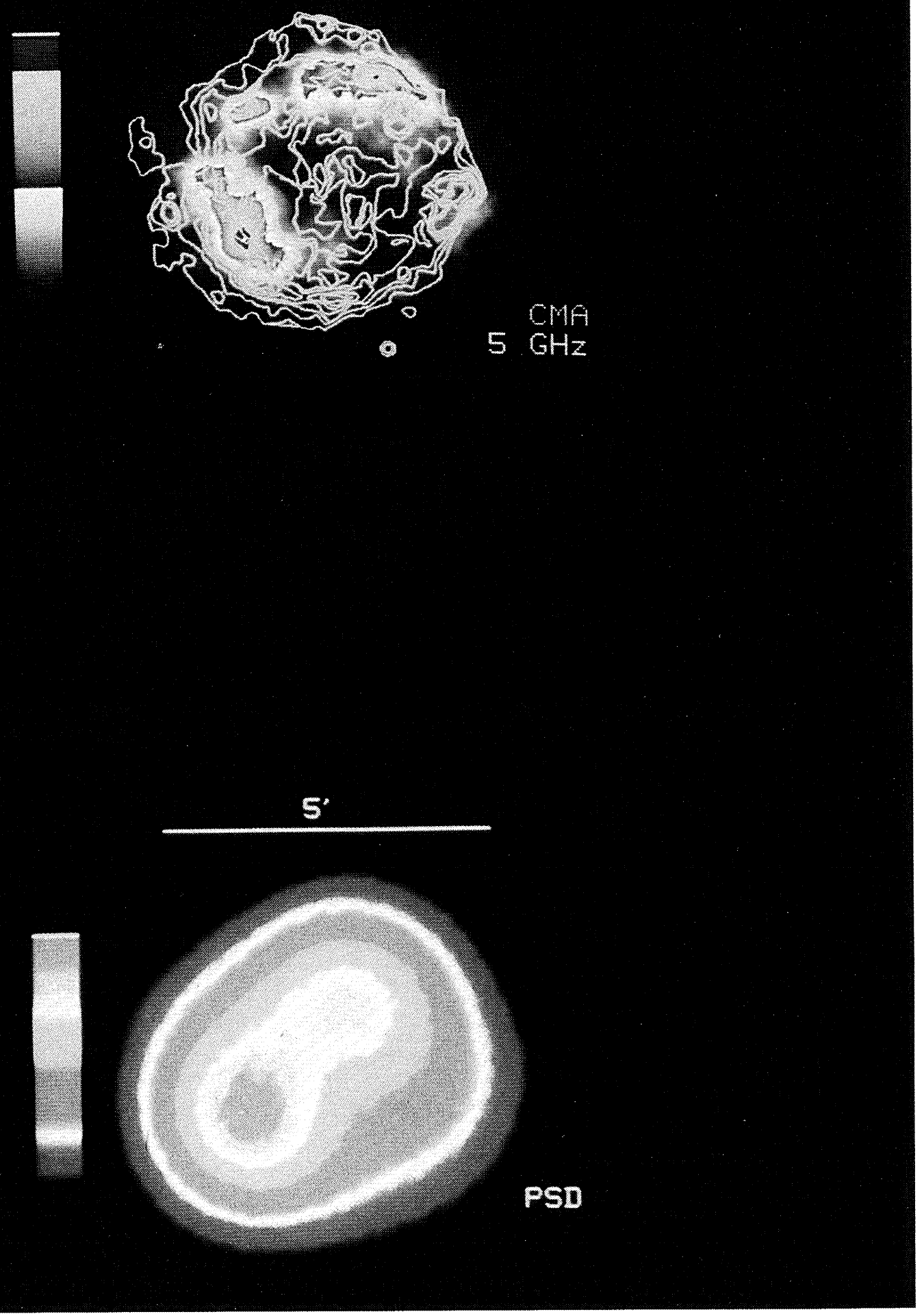

FIG. 3.-The top panel shows the EXOSAT image of Cas A obtained with the CMA detectors. Shown as an overlay is a $5 \mathrm{GHz}$ radio contour map which illustrates that, although the X-ray map and radio contours correlate very well, there is still significant X-ray emission outside the outermost radio contours. For comparison, the bottom panel shows the same image as obtained with the PSD detector, obtained by adding the images in nine spectral bands after convolving to equal spatial resolution.

JANSEN et al. (see 331,952)
(C) American Astronomical Society
- Provided by the NASA Astrophysics Data System 